\title{
Melatonin decreases cell proliferation, impairs myogenic differentiation and triggers apoptotic cell death in rhabdomyosarcoma cell lines
}

\author{
SILVIA CODENOTTI $^{1}$, MICHELA BATTISTELLI ${ }^{2}$, SABRINA BURATTINI $^{2}$, SARA SALUCCI $^{2}$, \\ ELISABETTA FALCIERI ${ }^{2}$, RITA REZZANI $^{3}$, FIORELLA FAGGI $^{1}$, MARINA COLOMBI $^{1}$, \\ EUGENIO MONTI ${ }^{1}$ and ALESSANDRO FANZANI ${ }^{1}$
}

\author{
${ }^{1}$ Department of Molecular and Translational Medicine, University of Brescia, Viale Europa 11, I-25123 Brescia; \\ ${ }^{2}$ Department of Earth, Life and Environmental Sciences (DiSTeVA), University Carlo Bo, I-61029 Urbino; \\ ${ }^{3}$ Department of Clinical and Experimental Sciences, University of Brescia, Viale Europa 11, I-25123 Brescia, Italy
}

Received January 14, 2015; Accepted March 30, 2015

DOI: $10.3892 /$ or.2015.3987

\begin{abstract}
Melatonin is a small indole produced by the pineal gland and other tissues, and has numerous functions that aid in the maintenance of the whole body homeostasis, ranging from the regulation of circadian rhythms and sleep to protection from oxidative stress. Melatonin has also been reported to counteract cell growth and chemoresistance in different types of cancer. In the present study, we investigated the effects of exogenous melatonin administration on different human cell lines and primary mouse tumor cultures of rhabdomyosarcoma (RMS), the most frequent soft tissue sarcoma affecting childhood. The results showed that melatonin significantly affected the behavior of RMS cells, leading to inhibition of cell proliferation and impairment of myogenic differentiation followed by increased apoptotic cell death, as observed by immunoblotting analysis of apoptosis-related markers including $\mathrm{Bax}, \mathrm{Bcl}-2$ and caspase-3. Similar findings were observed using a combination of microscopy techniques, including scanning/transmission electron and confocal microscopy. Furthermore, melatonin in combination with doxorubicin or cisplatin, two compounds commonly used for the treatment of solid tumors, increased the sensitivity of RMS cells to apoptosis. These data indicated that melatonin may be effective in counteracting RMS tumor growth and chemoresistance.
\end{abstract}

Correspondence to: Dr Alessandro Fanzani, Department of Molecular and Translational Medicine, University of Brescia, Viale Europa 11, I-25123 Brescia, Italy

E-mail: alessandro.fanzani@unibs.it

Abbreviations: FGFR4, fibroblast growth factor receptor 4; FOXO1, forkhead box O1; Myf6, myogenic factor 6; Pax3 or -7, paired box 3 or -7

Keywords: apoptosis, chemoresistance, melatonin, rhabdomyosarcoma

\section{Introduction}

Soft tissue sarcomas are tumors arising from mesenchymal cell precursors that are committed towards the morphogenesis of soft tissues such as fat, muscle and deep skin tissues. Rhabdomyosarcoma (RMS) is considered a myogenic tumor and is classified as the most frequent sarcoma affecting children and adolescents (1). The current classification defines five different histotypes, with embryonal (eRMS) and alveolar (aRMS) subsets being the most frequently observed in children $<5$ years and in adolescents, respectively $(2,3)$. The eRMS variant is the most treatable and most common subtype representing $\sim 80 \%$ of RMS, while aRMS is more aggressive and characterized by a poorer prognosis. The genetic alterations characterizing eRMS commonly involve the loss of heterozygosis on chromosome region 11p15.5 (4), gain of chromosomes $(5,6)$ and mutations on genes involved with growth factor signaling pathways (7-15). This leads to uncontrolled cell growth and the interruption of proper myogenic differentiation. Conversely, the aRMS subset is commonly characterized by the expression of Pax3-forkhead box O1 (FOXO1), a fused transcription factor derived from the chromosomal translocation $\mathrm{t}(2 ; 13)(\mathrm{q} 35 ; \mathrm{q} 14)$, which juxtaposes the DNA-binding domain of Pax 3 to the potent transactivation domain of FOXO1 (16). In the absence of the original Pax3 transactivation domain, the chimeric protein drives in a constitutive manner the transcription of numerous genes involved in muscle embryogenesis, such as $c-M E T$ and FGFR4, essentially maintaining the muscle precursors in a long-lasting proliferative state and thereby facilitating tumor initiation, aggressiveness and metastatic ability (16-19).

Melatonin is a small molecule derived from tryptophan metabolism and secreted by the pineal gland during periods of darkness $(20,21)$. Melatonin is involved in the regulation of seasonal and circadian rhythms, effects that are mediated by melatonin binding to specific receptors, such as MT1 and MT2, which in turn trigger the downstream activation of CLOCK and BMAL1 factors driving a complex transcriptional program $(22,23)$. Besides regulating the 
rhythm adaptations, melatonin exhibits additional functions, including the promotion of cell survival, neuroprotection and cardioprotection likely due to antioxidant properties (24-27). Notably, previous findings have shown a role for melatonin in preventing tumor initiation and progression (28-34). Specifically, melatonin was reported to inhibit cell proliferation and induce apoptosis in osteosarcoma (35), B-lymphoma (36) and colorectal cancer cells (37), as well as to decrease the weight of tumor masses in breast and prostate cancer (38-40). Based on those reports, the present study was conducted to examine the effects of melatonin on RMS cell lines in vitro. For this purpose, we employed human RMS cell lines as well as primary mouse tumor cultures established from transgenic mice (41) to evaluate the effects of melatonin on cell viability, proliferation and differentiation. In vitro assays and morphological analysis using electronic and confocal microscopy were performed.

\section{Materials and methods}

Reagents. Reagents were purchased from Sigma-Aldrich (Milan, Italy), unless otherwise stated. Cell culture materials were purchased from Jet-Biofil (Carlo Erba Reagents-Dasit Group, Cornaredo, Italy).

Human cell lines and primary mouse tumor cultures. Human RD (eRMS) and RH30 (aRMS) cells were purchased from the European Collection of Cell Cultures (ECACC; Salisbury, UK). The primary tumor mouse cultures, U57810 (eRMS) and U23674 (aRMS), were established from transgenic mice (41). In particular, eRMS mouse models were generated by crossing p53- or Ptch1-deficient conditional mice with Myf6-Cre mice to achieve the deletion of p53 with or without concurrent Ptch1 deletion in differentiating Myf6-positive myoblasts. The Myf6Cre/p53 $3^{-/-}$mouse strain was characterized by the highest percentage of eRMS (41). aRMS mouse models were characterized by knockout alleles of p53 or INK4a/ARF locus with concomitant Pax3-FOXO1 knock-in allele, which were restricted to differentiating Myf6-positive myoblasts, resulting in the Myf6Cre/Pax3-FOXO1/p53-1mouse strain (41).

Cell culture conditions. Cells were maintained at $37^{\circ} \mathrm{C}$ and $5 \% \mathrm{CO}_{2}$ in a humidified incubator and cultured in a growth medium (GM) comprising high-glucose Dulbecco's modified Eagle's medium (DMEM) (D6429) supplemented with $10 \%$ fetal bovine serum (FBS) (FA30A15101; Carlo Erba, Milan, Italy) in the presence of $100 \mu \mathrm{g} / \mathrm{ml}$ penicillin/streptomycin (A5955) and 1\% L-glutamine (G7513) (only for RH30 cells). To induce myodifferentiation, $80 \%$ confluent cells were switched to differentiation medium (DM) comprising DMEM supplemented with $2 \%$ horse serum (H1270).

Pharmacological treatments. Cells were treated with different concentrations $(0.01,0.1,1$, and $2 \mathrm{mM})$ of melatonin (461326) or vehicle alone [dimethylsulfoxide (DMSO)] (D5879). The cells were also treated with melatonin in the absence or presence of chemotherapeutic drugs such as doxorubicin $(0.15 \mathrm{ng} / \mathrm{ml})(\mathrm{D} 1515)$ and cisplatin $(2 \mu \mathrm{g} / \mathrm{ml})(\mathrm{P} 4394)$, which were previously diluted in $\mathrm{H}_{2} \mathrm{O}$ and DMSO, respectively.
Cell proliferation assay. eRMS and aRMS cells were seeded in 24-well plates at a density of $10 \times 10^{3}$ and $15 \times 10^{3}$, respectively. After 24,48 and $72 \mathrm{~h}$ of melatonin treatment, the cells were harvested, fixed in paraformaldehyde (F8775) and stained for 10 min with crystal violet (C0075) solution $[0.2 \%$ in phosphate-buffered saline (PBS) (D8537) with $20 \%$ methanol (32213)]. The samples were then collected in $600 \mu 1$ of SDS (74255) solution (1\% in PBS) and absorbance of the total homogenates, as measured by reading the plate at $540 \mathrm{~nm}$ emission wavelengths, was proportional to the amount of viable and proliferating cells that incorporated the crystal violet. In addition, the cell proliferation was expressed as the growth rate, which was calculated using Microsoft Excel 2010 software. The results were representative of at least three independent experiments.

Cell viability assay. A neutral red assay was employed to determine the percentage of viable cells that incorporated the neutral red dye in lysosomes, as initially described by Borenfreund and Puerner (42), a protocol subsequently modified by Repetto et al (43). Briefly, the cells were seeded in 96 -well plates at a density of $1.5 \times 10^{3}$. After 24,48 and $72 \mathrm{~h}$ of melatonin treatment, the cells were incubated for $2 \mathrm{~h}$ with neutral red dye (40 $\mu \mathrm{g} / \mathrm{ml})$ (N7005) dissolved in DMEM with $5 \%$ FBS. After washing the cells with PBS, $150 \mu 1$ of neutral red destaining solution [50\% ethanol (02860), 49\% deionized water, and $1 \%$ glacial acetic acid $(100015 \mathrm{~N}$; BDH Laboratory Supplies, Dawsonville, GA, USA)] was added, followed by gentle agitation for $10 \mathrm{~min}$, until complete dissolution was achieved. Absorbance was then measured by reading the plate at $540 \mathrm{~nm}$ emission wavelengths. The results were analyzed using Microsoft Excel 2010 software and presented as the percentage of control values. Images of cell viability assays showed representative results of at least three independent experiments.

Immunoblotting analysis. Protein homogenates were obtained by harvesting cells in a cold RIPA lysis buffer, comprising 20 mM Tris- $\mathrm{HCl}$ (pH 7.6) (T1503), 1\% Nonidet P40 (NP40), $0.5 \%$ sodium deoxycholate (D6750), $0.1 \%$ SDS (74255), $50 \mathrm{mM}$ $\mathrm{NaCl}$ (S7653) and a cocktail of protease inhibitors (1836153; Roche, Milan, Italy) plus phosphatase inhibitors [1 $\mathrm{mM} \mathrm{Na}_{3} \mathrm{VO}_{4}$ (S6508) and $4 \mathrm{mM} \mathrm{NaF} \mathrm{(S7920)].} \mathrm{The} \mathrm{protein} \mathrm{concentration}$ was calculated by a Bradford reagent (B6916) assay and an equal amount of protein samples was separated by SDS-PAGE under reducing conditions and transferred to polyvinylidene fluoride membranes (P2938). Incubation with specific primary antibodies was followed by peroxidase-conjugated secondary antibodies (goat polyclonal anti-mouse IgG-HRP sc-2005; from Santa Cruz Biotechnology, Inc., Dallas, TX, USA; donkey polyclonal anti-rabbit IgG no. 31458; from Thermo Scientific, Erembodegem, Belgium) and the resulting immune complexes were visualized using the enhanced chemiluminescence reagent (STS-E 500; GeneSpin, Milan, Italy). Immune-reactive bands were quantified using densitometry analyses (Software Gel-Pro Analyzer, version 4).

Antibodies. The antibodies were purchased from Santa Cruz Biotechnology, Inc., unless otherwise stated. The primary antibodies used were: mouse monoclonal anti-myosin heavy 

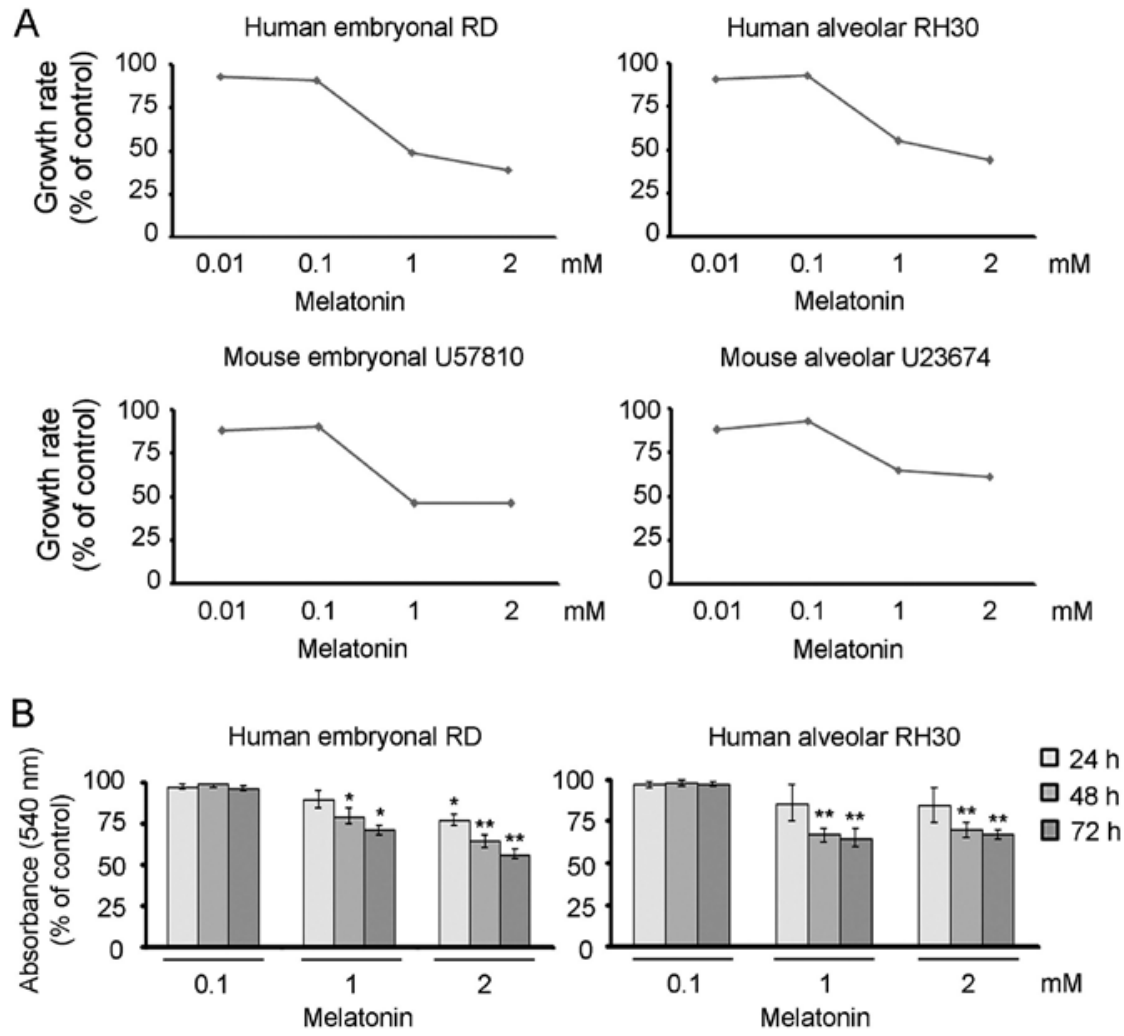

Figure 1. Effects of melatonin administration on the proliferation and viability of RMS cell lines. Crystal violet assay was employed to assess the proliferation of human cell lines (RD and RH30) and primary mouse tumor cultures (U57810 and U23674) in the absence or presence of increasing melatonin concentrations $(0.01,0.1,1$ and $2 \mathrm{mM}$ ) for up to 24,48 and $72 \mathrm{~h}$. (A) Line graphs show the growth rate, which was calculated by assessing the values obtained from 24 up to $72 \mathrm{~h}$. Results are representative of four independent experiments. " $\mathrm{P}<0.05 ;{ }^{* * *} \mathrm{P}<0.001 ;{ }^{* * * *} \mathrm{P}<0.0001$. (B) Cell viability of human RD and RH30 lines, treated with the indicated doses of melatonin for 24,48 and $72 \mathrm{~h}$, was assessed using the neutral red assay. Absorbance values reflect the amount of viable cells that have incorporated the neutral red dye. Results are representative of three independent experiments. ${ }^{*} \mathrm{P}<0.05 ;{ }^{* *} \mathrm{P}<0.001 ;{ }^{* * *} \mathrm{P}<0.0001$. RMS, rhabdomyosarcoma.

chain, (sc-32732; 1:1,000 dilution); mouse monoclonal anticaveolin-3 (610420; 1:1,000 dilution; BD, Buccinasco, Italy); rabbit polyclonal anti-caspase-3 (H-277) (sc-7148; 1:500 dilution; Cell Signaling, Milan, Italy); rabbit polyclonal anti-Bax (sc-526; 1:500 dilution); rabbit polyclonal anti-Bcl-2 (sc-492; 1:500 dilution); and mouse monoclonal anti- $\alpha$-tubulin (T5168; 1:10,000 dilution; Sigma-Aldrich).

Scanning electron microscopy (SEM). RH30 cells were cultured and treated directly on coverslips in Petri dishes. After washing with $0.1 \mathrm{M}$ phosphate buffer, adherent and suspended cells were fixed with $2.5 \%$ glutaraldehyde (G5882) in $0.1 \mathrm{M}$ phosphate buffer for $1 \mathrm{~h}$. The suspended cells adhered to polylysine-coated coverslips. The samples were post-fixed with $1 \%$ osmium tetroxide $\left(\mathrm{OsO}_{4}\right)(\mathrm{O} 021$; Strumenti, Roma, Italy) in $0.1 \mathrm{M}$ phosphate buffer for $1 \mathrm{~h}$. After alcohol dehydration, the samples were critical point dried, gold sputtered and observed using a Philips 515 scanning electron microscope (FEI, Italy) (44).

Transmission electron microscopy (TEM). RH30-treated cells were washed and fixed with $2.5 \%$ glutaraldehyde (G5882) in $0.1 \mathrm{M}$ phosphate buffer for $15 \mathrm{~min}$. The cells were scraped and centrifuged at $300 \mathrm{x}$ g for $10 \mathrm{~min}$. The pellets were fixed in $2.5 \%$ glutaraldehyde for an additional $30 \mathrm{~min}$. The suspended cells were collected in Eppendorf, centrifuged and fixed for $45 \mathrm{~min}$ in glutaraldehyde. The samples were post-fixed in $1 \%$
$\mathrm{OsO}_{4}(\mathrm{O} 021)$ for $1 \mathrm{~h}$, alcohol dehydrated and embedded in araldite (02860) (45). Thin sections were stained with uranyl acetate and lead citrate and analyzed using a Philips CM10 transmission electron microscope (FEI).

Confocal microscopy fluorescence. Adherent cells were cultured and treated directly on coverslips in Petri dishes. The suspended cells were collected in Eppendorf, fixed in $4 \%$ paraformaldehyde (F8775) for $30 \mathrm{~min}$ and then plated on polylysine-coated coverslips. The cells were then fixed in $4 \%$ paraformaldehyde in PBS ( $\mathrm{pH} \mathrm{7.4)} \mathrm{for} 30 \mathrm{~min}$ and washed twice using PBS. The cells were then pre-treated with RNase A (10 $\mu \mathrm{g} / \mathrm{ml})$ (12091-021) in PBS for $30 \mathrm{~min}$ and exposed to an equal mixture of propidium iodide (PI; $1 \mu \mathrm{g} / \mathrm{ml})$ (P3566) and acridine orange (AO; $1 \mu \mathrm{g} / \mathrm{ml}$ ) (A3568) (all from Life Technologies, Monza, Italy) in PBS at room temperature in the dark for $10 \mathrm{~min}$.

$\mathrm{AO}$ and PI are intercalating fluorochromes that emit green and red fluorescence, respectively, when they are bound to DNA. Only AO diffuses through the plasma membrane of both viable and early apoptotic cells. Viable cells exhibit a green nucleus with intact structure, while apoptotic cells exhibit a bright-green nucleus, with condensation of chromatin. PI only enters late apoptotic and necrotic cells, resulting in double staining with AO and PI (45). The samples were observed with a Leica TCS-SP5 CLSM connected to a DMI 6000 CS inverted microscope (Leica Microsystems CMS GmbH; 
$\mathrm{AO}$ and PI excitation were at 488 and $500 \mathrm{~nm}$, respectively, and their emission signals were detected at 617 and $525 \mathrm{~nm}$, respectively).

Statistical analysis. The differences between the groups were analyzed by the unpaired Student's t-test and one-way ANOVA test (with Dunnet's post-hoc test), using Prism 4 software for Windows (GraphPad Software, San Diego, CA, USA). P $<0.05$ was considered to indicate a significant result.

\section{Results}

Melatonin suppresses cell proliferation and triggers apoptotic and necrotic features in RMS cells. We evaluated whether melatonin administration would influence the cell growth of the human RMS cell lines (i.e. embryonal RD and alveolar RH30) and primary mouse tumor cultures (i.e., embryonal U57810 and alveolar U23674). For this purpose, the proliferation of cells that received melatonin once was determined over a time-course of $72 \mathrm{~h}$ by means of crystal violet assay. Treating different lines with increasing concentrations of melatonin, ranging from 0.01 to $2 \mathrm{mM}$, led to a significant impairment of cell proliferation starting from a dose of $1 \mathrm{mM}$ in comparison to vehicle-treated cells, as indicated after calculation of the growth rate (Fig. 1A). To determine whether the melatonin effects were attributable to the inhibition of cell proliferation rather than impaired cell viability, we performed the neutral red assay using the cell lines under the same experimental conditions. As shown in Fig. 1B, $72 \mathrm{~h}$ of exposure with a concentration of 1 or $2 \mathrm{mM}$ triggered the loss of $\sim 50 \%$ of RMS cells in comparison to vehicle-treated cells, indicating that melatonin has cytotoxic effects. This latter result was confirmed by immunoblotting analysis of Bax and Bcl-2 expression, two proteins that can be either pro- or anti-apoptotic, respectively (46-48).

As shown in Fig. 2, treatment of RD and RH30 cells with $1 \mathrm{mM}$ melatonin promoted an increase in the pro-apoptotic Bax expression, while the expression levels of anti-apoptotic $\mathrm{Bcl}-2$ were downregulated in comparison to those in untreated cells. These results indicated that melatonin, not only behaves as a cytostatic factor on RMS cell growth, but also impairs the survival of different RMS lines by triggering an apoptotic program. Thus, at the ultrastructural level, the pro-apoptotic effects of melatonin, RH30 cells treated or untreated were analyzed by electronic and confocal microscopy.

Control cells showed an obvious healthy morphology characterized by the presence of intact subcellular structures, as observed by means of SEM (Fig. 3A) and TEM (Fig. 3B and C), respectively. In addition, AO/PI double staining showed a uniform green labeling suggestive of cellular healthy structures (Fig. 3D). After $1 \mathrm{mM}$ melatonin for $24 \mathrm{~h}$ a heterogeneous situation developed: some cells maintained good cell viability similar to the control condition, while other cells showed a round apoptotic-like morphology (Fig. 3E and F). In particular, TEM analyses revealed some cells with an intense chromatin condensation, a typical apoptotic pattern (Fig. 3G). At the confocal microscopy level some cells appeared rounded and early apoptotic features were evident (Fig. 3H). After $48 \mathrm{~h}$, melatonin-treated cells were almost all detached showing a round apoptotic morphology while only a small number of

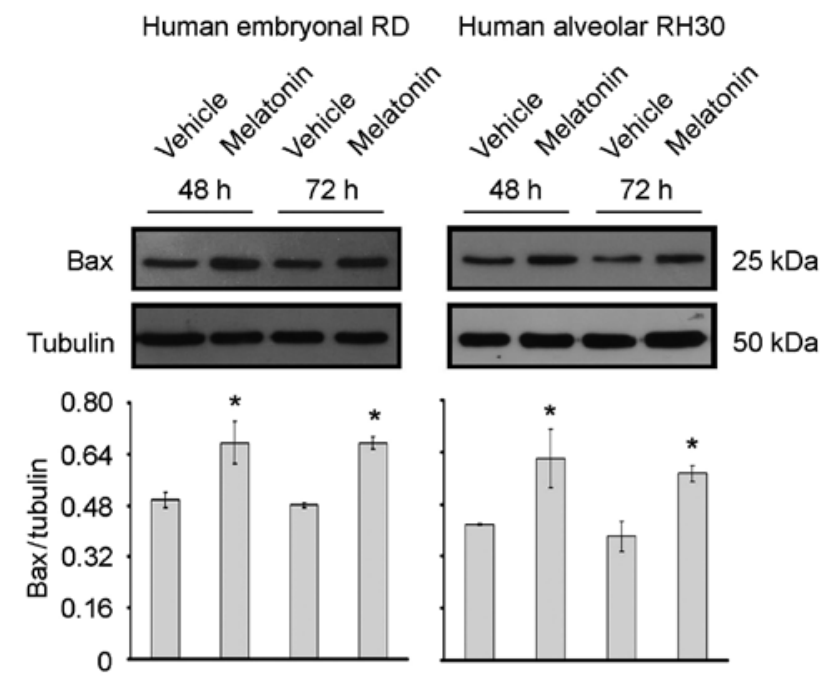

Human embryonal RD Human alveolar RH30
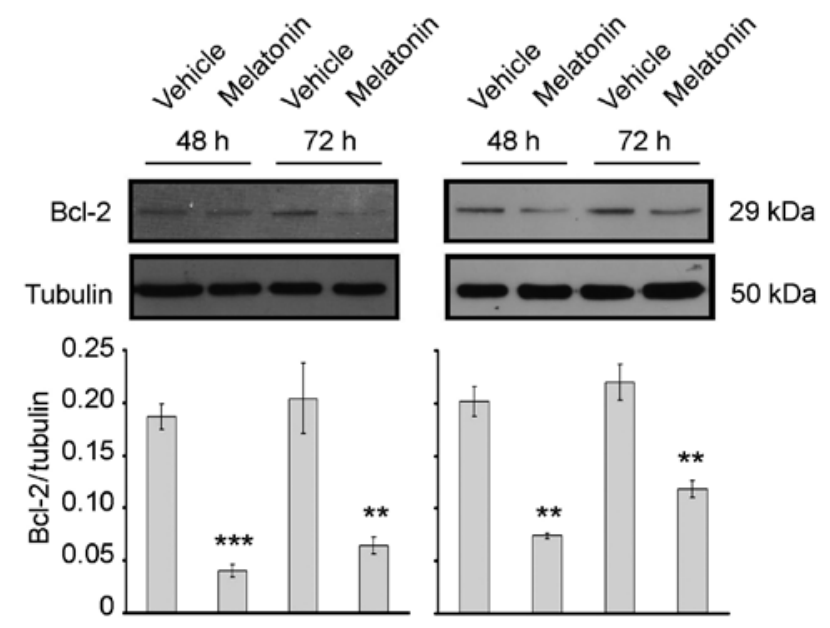

Figure 2. Analysis of apoptosis-related markers in melatonin-treated RMS cells. Proteins levels of Bax and Bcl-2 were evaluated using immunoblotting analysis in RD and RH30 lines treated with $1 \mathrm{mM}$ melatonin or vehicle for 48 and $72 \mathrm{~h}$. Protein bands were quantified after normalization with respect to tubulin. ${ }^{*} \mathrm{P}<0.05 ;{ }^{* *} \mathrm{P}<0.001 ;{ }^{* * * *} \mathrm{P}<0.0001$. RMS, rhabdomyosarcoma.

adherent cells exhibited an atrophic behavior, due to cytoplasm shrinkage (Fig. 3I). In addition, rounded cells suggestive of necrotic features also appeared (Fig. 3J). These cells were characterized by typical apoptotic features, including the presence of chromatin condensation, cytoplasm vacuolization and secondary necrosis as confirmed by TEM analysis (Fig. 3K). Consistent with this, an increased number of apoptotic cells showed orange areas due to PI permeability suggestive of cells in late apoptosis (Fig. 3L). After $72 \mathrm{~h}$, the melatonin-treated cells observed at the SEM level exhibited apoptotic and necrotic features, being completely detached and showing a rounded morphology, with disruption of cell membranes in those that were necrotic (Fig. 3M and N). As shown by TEM, necrotic cells were characterized by cytoplasmic vacuolization due to membrane disruption and loss of cell components during the necrotic process (Fig. 3Q). The few adherent apoptotic cells exhibited large orange areas (Fig. 3P, inset) as observed during late apoptosis, whereas suspended cells showed bright-green nuclei predictive of apoptotic bodies (Fig. 3P). RH30 cells treated with $2 \mathrm{mM}$ melatonin were characterized 


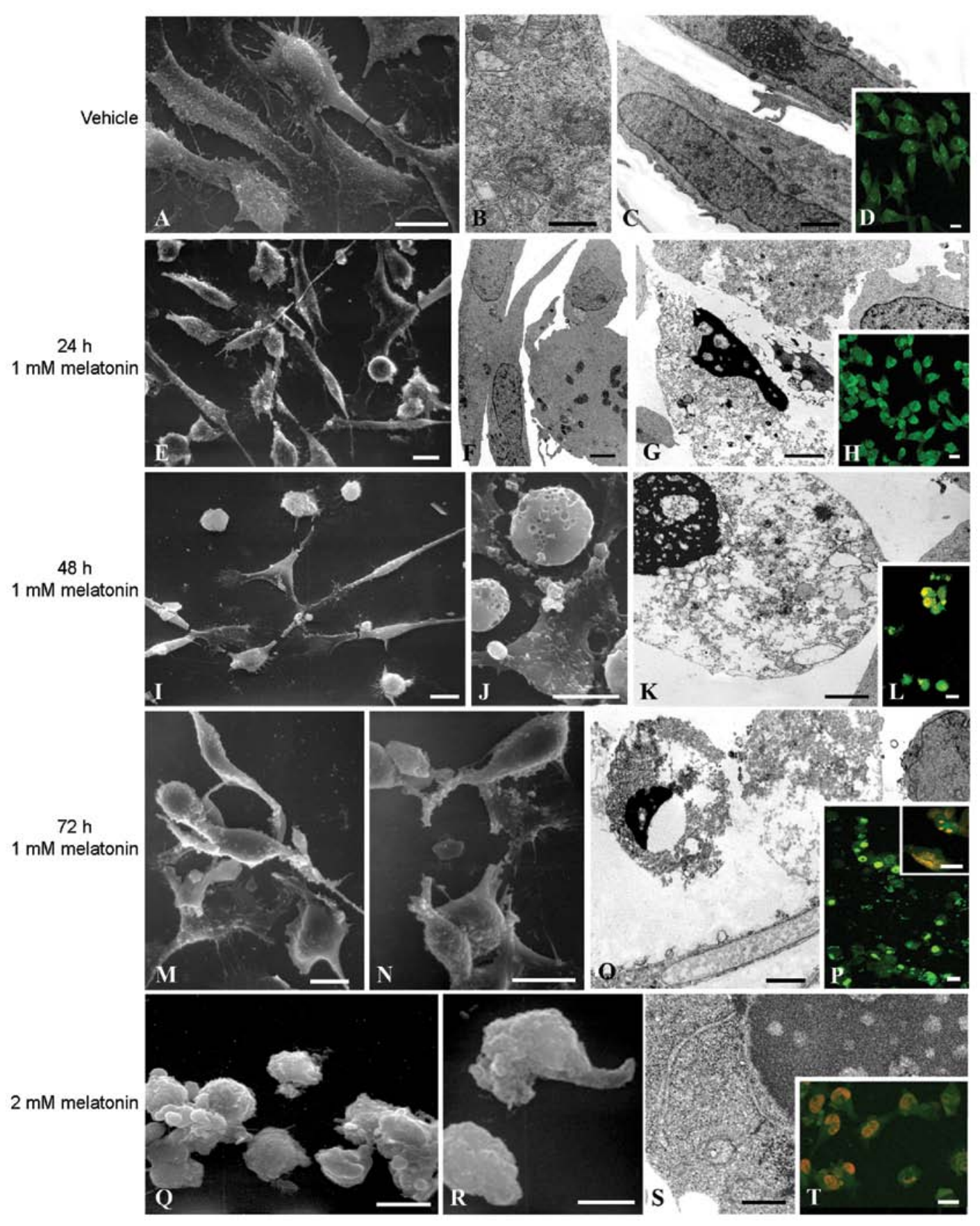

Figure 3. Microscopy analysis of the morphological changes occurring in melatonin-treated RH30 cells. Untreated control cells were observed using (A) SEM, (B and C) TEM and (D) CLSM. (A) Control cells showed adherent morphology. (B) Mitochondrion with healthy structure. (C) Well-defined intact nucleus. (E) AO green staining of vital cells. Bars, A and D, 10 micron; C, 2 micron; and B, $500 \mathrm{~nm}$. Cytotoxic effect of melatonin on RH30 cells. RH30 cells were treated with melatonin at a concentration of $1 \mathrm{mM}$ for 24,48 and $72 \mathrm{~h}$ and observed at (E, I, J, M and N) SEM, (F, G, K and O) TEM and (H, L and P) CLSM. (E) RH30-treated cells with an elongated morphology. (F) Intact nucleus of a vital cell. (G) Adherent cell showing an apoptotic body. (H) AO green staining of adherent cells. (I) Adherent cells with an elongated morphology with some apoptotic rounded cells. (J) Rounded apoptotic cells. (K) Apoptotic cells showing an apoptotic body and a well-defined plasma membrane. (L) Orange staining due to PI permeability in apoptotic cells. (M and N) Necrotic cells with disrupted cell membranes. (O) Necrotic cells showing disruption of cell membranes and cytoplasmic vacuolization. (P) AO bright-green staining in early apoptotic cells and PI orange staining in late apoptotic and necrotic cells. Bars: E, H, I, J, L, M, N and P, 10 micron; F, G, K and O, $500 \mathrm{~nm}$. Cytotoxic effect of melatonin on RH30 cells. RH30 cells were treated with $2 \mathrm{mM}$ melatonin for 24 and $48 \mathrm{~h}$ and observed at (Q and R) SEM, (S) TEM and (T) CLSM. (Q and R) Necrotictreated cells with disrupted cell membranes. (S) Necrotic cell showing DNA condensation and disruption of cell membranes. (T) PI orange staining in apoptotic and necrotic cells. Bars: Q, R and T, 10 micron; S, $500 \mathrm{~nm}$. SEM, scanning electron microscopy; TEM, transmission electron microscopy; AO, acridine orange; PI, propidium iodide.

by round, blebbed cells after 24 and $48 \mathrm{~h}$ (Fig. 3Q and R, respectively). TEM analysis at $48 \mathrm{~h}$ of treatment highlighted dark areas predictive of DNA condensation and cytoplasmic vacuolization (Fig. 3S). Using AO and PI double-staining, melatonin-treated cells showed orange staining at the nuclei due to late apoptosis and necrosis already after $24 \mathrm{~h}$ (Fig. 3T), as also observed after $48 \mathrm{~h}$ (data not shown).

Melatonin impairs the myogenic differentiation in embryonal $R D$ cells. Forced differentiation of tumor cells induced by 
A

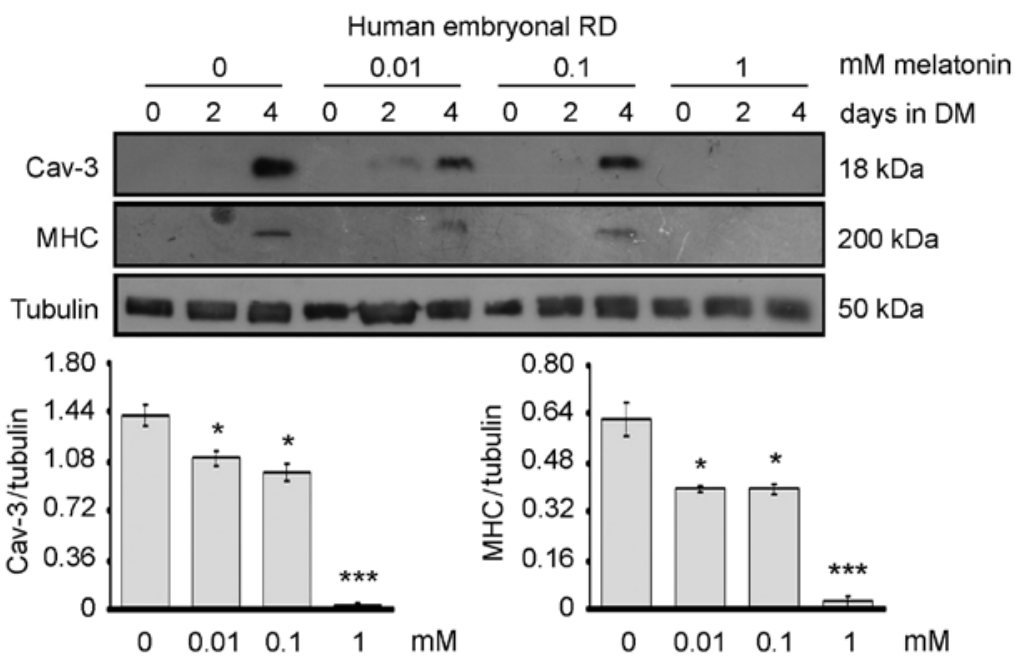

B

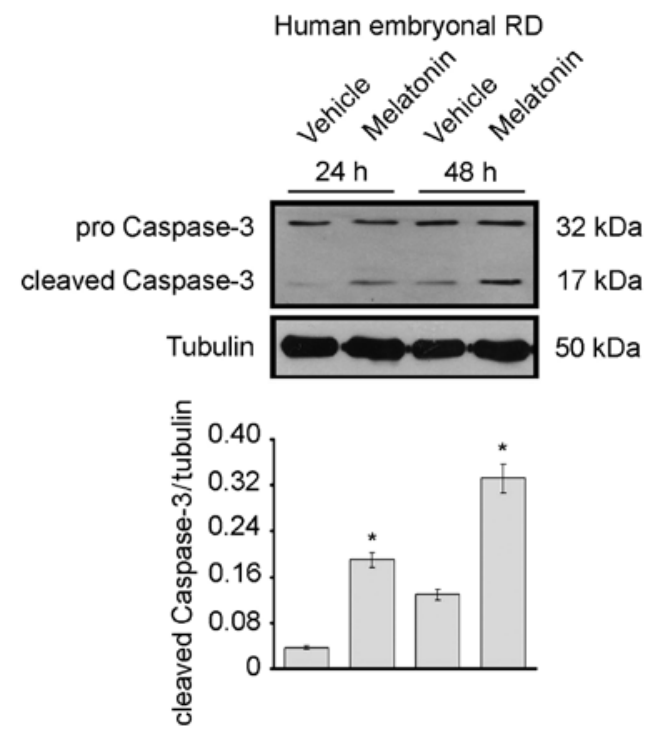

Figure 4. Expression analysis of differentiation markers in melatonin-treated RMS cells. (A) Expression levels of Cav-3 and MHC were analyzed using immunoblotting analysis in RD cells cultured in DM for the indicated time-points in the presence or absence of increasing melatonin concentrations. Protein bands were quantified after normalization with respect to tubulin. ${ }^{*} \mathrm{P}<0.05 ;{ }^{* * *} \mathrm{P}<0.0001$. (B) Expression levels of cleaved caspase-3 fragments in differentiated RD cells after 24 and 48 h of treatment with $1 \mathrm{mM}$ melatonin were analyzed by western blot analysis. Protein bands were quantified after normalization with respect to tubulin. ${ }^{\mathrm{P}}<0.05$. RMS, rhabdomyosarcoma; Cav-3, caveolin-3; MHC, myosin heavy chain; DM, differentiation medium.

anticancer agents has been widely exploited to limit the growth of tumor masses. In this regard, melatonin has been hypothesized to promote a differentiated phenotype in some tumors, such as gastric (49) and prostate cancer (50). Thus, to understand whether melatonin influences the myogenic differentiation of RMS, we employed human RD cells, which commonly exhibit a consistent myogenic potential in comparison to alveolar RH30 cells. For RD cells, the DM in the absence or presence of melatonin was replaced daily using different concentrations of melatonin. The extent of myogenic differentiation reached by cells in the different conditions was measured by immunoblotting analysis of markers that are normally increased during the differentiation of myoblasts, including caveolin-3 (Cav-3) and myosin heavy chain (MHC). Melatonin treatment led to a dose-dependent impairment of myogenic differentiation, since Cav-3 and MHC levels were reduced in comparison to the controls. In particular, melatonin at a concentration of $1 \mathrm{mM}$ completely abolished the myogenic differentiation in RMS cells, as both Cav-3 and MHC levels were undetectable (Fig. 4A). We also administered melatonin to RD cells after which they were differentiated for 4 days and then analyzed for caspase-3 proteolytic activation, which is commonly utilized as a readout of the cell apoptotic program. As observed by immunoblotting analysis, melatonin-treated differentiated RD cells were characterized by increased levels of caspase-3 cleaved fragments ( 19 and $17 \mathrm{kDa})$ in comparison to vehicle-treated cells after treatment for 24 and $48 \mathrm{~h}$ (Fig. 4B). These experiments demonstrated that melatonin has no positive effects on RMS differentiation, but behaves as a cytotoxic drug by triggering a caspase-dependent apoptosis.

Melatonin sensitizes RD and RH3O cells to cell death induced by chemotherapeutic agents doxorubicin and cisplatin. Previous findings have suggested that combination therapies including melatonin and conventional cancer drugs enhance success by increasing drug efficacy while reducing their side effects. In most clinical trials where melatonin was used in conjunction with chemotherapeutic drugs, improved overall 

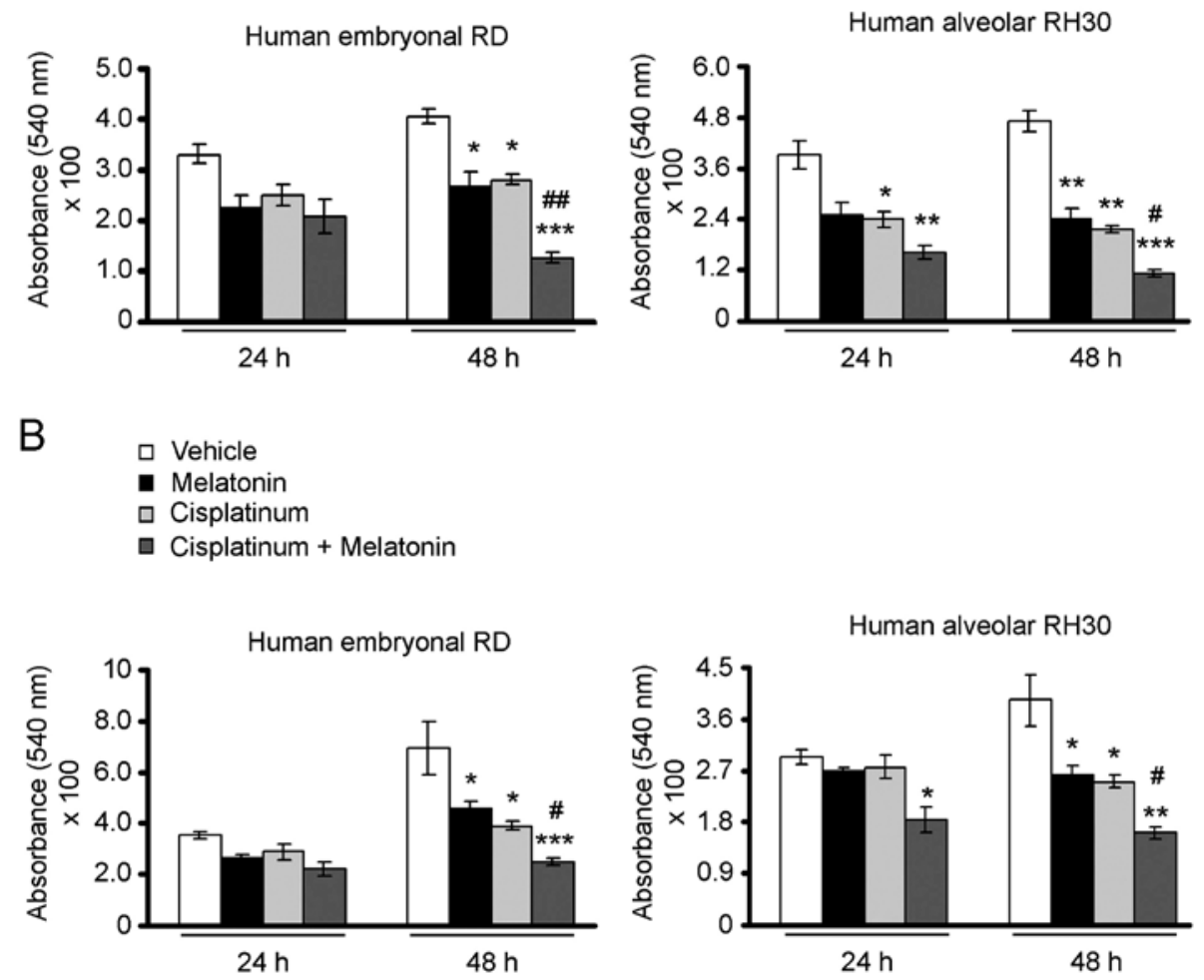

Figure 5. Viability of RMS cells after the combined treatment with melatonin and chemotherapeutic drugs. Crystal violet assay was employed to evaluate the viability of RD and RH30 lines following combined treatment with melatonin and doxorubicin or cisplatin for 24 and 48 h. (A) Cells were either treated with $1 \mathrm{mM}$ melatonin or $0.15 \mathrm{ng} / \mathrm{ml}$ doxorubicin or a combination of the two. Histograms show the absorbance values reflecting the amount of viable cells that have incorporated the crystal violet. (B) Cells were treated with $1 \mathrm{mM}$ melatonin or $2 \mu \mathrm{g} / \mathrm{ml}$ cisplatin or a combination of the two. The histograms show absorbance values reflecting the amount of viable cells that incorporated the crystal violet. Results are representative of four independent experiments. ${ }^{*} \mathrm{P}<0.05 ;{ }^{* *} \mathrm{P}<0.001$;

${ }^{* * *} \mathrm{P}<0.0001$ vs. untreated cells, ${ }^{\#} \mathrm{P}<0.05 ;{ }^{\# \#} \mathrm{P}<0.001$ vs. cells treated with melatonin or doxorubicin or cisplatin alone. RMS, rhabdomyosarcoma.

survival and patient conditions were observed $(51,52)$. This suggests that melatonin enhances the efficacy of chemotherapy and reduces side effects $(53,54)$. A combination of melatonin and doxorubicin was reported to enhance the growth inhibitory effect and induction of apoptosis in human hepatoma cells in comparison to melatonin or doxorubicin used alone (55). To verify the combined effects of melatonin and chemotherapy drugs on RMS chemoresistance, RD and RH30 cells were concurrently treated with $1 \mathrm{mM}$ melatonin and $0.15 \mathrm{ng} / \mathrm{ml}$ doxorubicin or $2 \mu \mathrm{g} / \mathrm{ml}$ cisplatin for up to $48 \mathrm{~h}$. As shown in Fig. 5, the two chemotherapies were effective in reducing the proliferation of RMS cell lines in comparison to the controls, as observed after $48 \mathrm{~h}$ of treatment. In addition, the concomitant treatment of melatonin with doxorubicin for up to $48 \mathrm{~h}$ produced a synergistic inhibitory effect (Fig. 5A). Similar results were obtained following treatment with melatonin and cisplatin (Fig. 5B). These results demonstrate a possible role of melatonin in improving the chemotherapy efficacy.

\section{Discussion}

Results obtained in the present study indicate that melatonin, when used at concentrations varying from 0.01 to $2 \mathrm{mM}$, profoundly affect the cell survival of rhabdomyosarcoma
(RMS), the most frequent myogenic sarcoma affecting children and adolescents (4). In human cell lines representative of the most frequent RMS categories, i.e., the eRMS and aRMS subtypes, we observed that melatonin limited cell proliferation and triggered morphological and subcellular changes typically recognizable in apoptotic cells, such as DNA fragmentation, disruption of cell membranes and proteolytic cleavage of caspases. We also observed similar effects in primary mouse tumor RMS cultures which, having been derived from mice with specific genetic backgrounds, faithfully recapitulate the onset of RMS genesis (41). In the cell cultures, the ability of melatonin to increase apoptosis was not exclusively correlated with cell cycle-dependent effects, since we observed melatonin to be effective in triggering cell death even in RMS cells that had withdrawn from the cell cycle to attempt differentiation. These observations suggest a potential efficacy of melatonin towards undifferentiated and more differentiated tumor histotypes. Studies on cancer cells have shown that antiproliferative and pro-apoptotic effects of melatonin were achieved with high doses, as compared with those detected in the blood at night. However, it is known that the intracellular levels of melatonin may be much higher than in blood (56). To explain the reason for melatonin often requiring to be added at pharmacological concentrations to produce inhibitory effects, a regulatory 
mechanisms by which its accumulation in cell membranes acts as a reservoir, limiting the net amount of the biological active indoleamine has been suggested (57).

Previous findings have indicated that melatonin produces no consistent adverse effects over various concentrations (58), suggesting that it may be useful to improve the efficacy of conventional cytotoxic agents. In this regard, we showed that melatonin synergized with chemotherapeutic drugs in human RMS cell lines. To the best of our knowledge, this is the first study showing indolamine to be effective in the enhancement of cell death on myogenic tumor cells using a complementary approach with doxorubicin or cisplatin drugs, as already observed in clinical trials on different types of cancer $(51-53,59)$.

The mechanisms underlying the effect of melatonin on apoptosis have not been clarified and appear to be, to some extent, context-specific (60-62). Some melatonin actions are mediated by specific membrane receptors, known as MT1 and MT2, that are known to be expressed in RMS tumor samples as demonstrated using tissue microarray analysis (data not shown). However, high doses of melatonin were found to be effective in inhibiting proliferation. Thus, RMS cells likely have a non-receptor-mediated action, since melatonin has been shown to even permeate into cells by means of receptor-independent processes. Notably, differentiated RD cells seemingly exhibited a marked responsiveness to melatonin, since already at 0.01 and $0.1 \mathrm{mM}$ concentrations we observed a negative effect on myogenic differentiation. In this context, whether the expression levels of melatonin receptors may differ between proliferating and differentiated cells, thus accounting for the different observed sensitivities should be investigated. Whereas in normal cells melatonin and its metabolites act as efficient radical scavengers (63), it has been suggested that changes in the oxidative status account for the ability of melatonin to induce apoptosis in cancer cells $(62,64,65)$. In this regard, a correlation between the increase in ROS production and the induction of melatonin-driven apoptosis has been reported in several cell lines (66). Consistent with this evidence, we have preliminarily observed a reduction in the melatonin cytotoxic effect by pretreating RMS cells with vitamin E (data not shown), a lipid-soluble antioxidant molecule. Although these observations are under investigation, it remains to be established whether the potential changes in the redox status is the cause rather than the consequence of the increased cell death.

In conclusion, the molecular mechanisms underlying the cytotoxicity on RMS cells, as observed for other types of cancer, deserve attention for establishing whether a rationale occurs for the introduction of melatonin as an adjuvant in the multimodality approach currently used against RMS.

\section{Acknowledgements}

We are grateful to Charles Keller (Oregon Health and Science University, USA) for providing the primary mouse tumor cultures of RMS.

\section{References}

1. Saab R, Spunt SL and Skapek SX: Myogenesis and rhabdomyosarcoma the Jekyll and Hyde of skeletal muscle. Curr Top Dev Biol 94: 197-234, 2011.

2. Dasgupta R and Rodeberg DA: Update on rhabdomyosarcoma. Semin Pediatr Surg 21: 68-78, 2012.
3. Parham DM, Alaggio R and Coffin CM: Myogenic tumors in children and adolescents. Pediatr Dev Pathol 15 (Suppl 1): S211-S238, 2012

4. Ognjanovic S, Linabery AM, Charbonneau B and Ross JA: Trends in childhood rhabdomyosarcoma incidence and survival in the United States, 1975-2005. Cancer 115: 4218-4226, 2009.

5. Williamson D, Missiaglia E, de Reyniès A, Pierron G, Thuille B, Palenzuela G, Thway K, Orbach D, Laé M, Fréneaux P, et al: Fusion gene-negative alveolar rhabdomyosarcoma is clinically and molecularly indistinguishable from embryonal rhabdomyosarcoma. J Clin Oncol 28: 2151-2158, 2010.

6. Chen X, Stewart E, Shelat AA, Qu C, Bahrami A, Hatley M, Wu G, Bradley C, McEvoy J, Pappo A, et al; St. Jude Children's Research Hospital-Washington University Pediatric Cancer Genome Project: Targeting oxidative stress in embryonal rhabdomyosarcoma. Cancer Cell 24: 710-724, 2013.

7. Shukla N, Ameur N, Yilmaz I, Nafa K, Lau CY, Marchetti A, Borsu L, Barr FG and Ladanyi M: Oncogene mutation profiling of pediatric solid tumors reveals significant subsets of embryonal rhabdomyosarcoma and neuroblastoma with mutated genes in growth signaling pathways. Clin Cancer Res 18: 748-757, 2012.

8. Shern JF, Chen L, Chmielecki J, Wei JS, Patidar R, Rosenberg M, Ambrogio L, Auclair D, Wang J, Song YK, et al: Comprehensive genomic analysis of rhabdomyosarcoma reveals a landscape of alterations affecting a common genetic axis in fusion-positive and fusion-negative tumors. Cancer Discov 4: 216-231, 2014.

9. Abraham J, Prajapati SI, Nishijo K, Schaffer BS, Taniguchi E, Kilcoyne A, McCleish AT, Nelon LD, Giles FG, Efstratiadis A, et al: Evasion mechanisms to Igf1r inhibition in rhabdomyosarcoma. Mol Cancer Ther 10: 697-707, 2011.

10. Taylor JG VI, Cheuk AT, Tsang PS, Chung JY, Song YK, Desai K, Yu Y, Chen QR, Shah K, Youngblood V, et al: Identification of FGFR4-activating mutations in human rhabdomyosarcomas that promote metastasis in xenotransplanted models. J Clin Invest 119: 3395-3407, 2009.

11. Crose LE and Linardic CM: Receptor tyrosine kinases as therapeutic targets in rhabdomyosarcoma. Sarcoma 2011: 756982, 2011.

12. Lee Y, Kawagoe R, Sasai K, Li Y, Russell HR, Curran T and McKinnon PJ: Loss of suppressor-of-fused function promotes tumorigenesis. Oncogene 26: 6442-6447, 2007.

13. Petricoin EF III, Espina V, Araujo RP, Midura B, Yeung C, Wan X, Eichler GS, Johann DJ Jr, Qualman S, Tsokos M, et al: Phosphoprotein pathway mapping: Akt/mammalian target of rapamycin activation is negatively associated with childhood rhabdomyosarcoma survival. Cancer Res 67: 3431-3440, 2007.

14. Guenther MK, Graab U and Fulda S: Synthetic lethal interaction between PI3K/Akt/mTOR and Ras/MEK/ERK pathway inhibition in rhabdomyosarcoma. Cancer Lett 337: 200-209, 2013.

15. Hahn H, Wojnowski L, Specht K, Kappler R, Calzada-Wack J, PotterD,Zimmer A, Müller U,Samson E, Quintanilla-Martinez L, et al: Patched target Igf2 is indispensable for the formation of medulloblastoma and rhabdomyosarcoma. J Biol Chem 275: 28341-28344, 2000.

16. Marshall AD and Grosveld GC: Alveolar rhabdomyosarcomaThe molecular drivers of PAX3/7-FOXO1-induced tumorigenesis. Skelet Muscle 2: 25, 2012.

17. Barr FG, Galili N, Holick J, Biegel JA, Rovera G and Emanuel BS: Rearrangement of the PAX3 paired box gene in the paediatric solid tumour alveolar rhabdomyosarcoma. Nat Genet 3: 113-117, 1993.

18. Graf Finckenstein F, Shahbazian V, Davicioni E, Ren YX and Anderson MJ: PAX-FKHR function as pangenes by simultaneously inducing and inhibiting myogenesis. Oncogene 27: 2004-2014, 2008.

19. Keller C and Guttridge DC: Mechanisms of impaired differentiation in rhabdomyosarcoma. FEBS J 280: 4323-4334, 2013.

20. Carlberg C: Gene regulation by melatonin. Ann NY Acad Sci 917: 387-396, 2000.

21. Stehle JH, Saade A, Rawashdeh O, Ackermann K, Jilg A, Sebestény T and Maronde E: A survey of molecular details in the human pineal gland in the light of phylogeny, structure, function and chronobiological diseases. J Pineal Res 51: 17-43, 2011.

22. Hardeland R: Melatonin: Signaling mechanisms of a pleiotropic agent. Biofactors 35: 183-192, 2009.

23. Challet E: Minireview: Entrainment of the suprachiasmatic clockwork in diurnal and nocturnal mammals. Endocrinology 148: 5648-5655, 2007.

24. Luchetti F, Betti M, Canonico B, Arcangeletti M, Ferri P, Galli F and Papa S: ERK MAPK activation mediates the antiapoptotic signaling of melatonin in UVB-stressed U937 cells. Free Radic Biol Med 46: 339-351, 2009. 
25. Luchetti F, Canonico B, Betti M, Arcangeletti M, Pilolli F, Piroddi M, Canesi L, Papa S and Galli F: Melatonin signaling and cell protection function. FASEB J 24: 3603-3624, 2010.

26. Tengattini S, Reiter RJ, Tan DX, Terron MP, Rodella LF and Rezzani R: Cardiovascular diseases: Protective effects of melatonin. J Pineal Res 44: 16-25, 2008.

27. Zhang HM and Zhang Y: Melatonin: A well-documented antioxidant with conditional pro-oxidant actions. J Pineal Res 57: 131-146, 2014

28. Bukowska A: Anticarcinogenic role of melatonin - potential mechanisms. Med Pr 62: 425-434, 2011 (In Polish).

29. Hrushesky WJ, Grutsch J, Wood P, Yang X, Oh EY, Ansell C, Kidder S, Ferrans C, Quiton DF, Reynolds J, et al: Circadian clock manipulation for cancer prevention and control and the relief of cancer symptoms. Integr Cancer Ther 8: 387-397, 2009.

30. Mao L, Yuan L, Slakey LM, Jones FE, Burow ME and Hill SM Inhibition of breast cancer cell invasion by melatonin is mediated through regulation of the p38 mitogen-activated protein kinase signaling pathway. Breast Cancer Res 12: R107, 2010.

31. Mediavilla MD, Sanchez-Barcelo EJ, Tan DX, Manchester L and Reiter RJ: Basic mechanisms involved in the anti-cancer effects of melatonin. Curr Med Chem 17: 4462-4481, 2010.

32. Santoro R, Marani M, Blandino G, Muti P and Strano S: Melatonin triggers p53Ser phosphorylation and prevents DNA damage accumulation. Oncogene 31: 2931-2942, 2012.

33. Schernhammer ES, Razavi P, Li TY, Qureshi AA and Han J: Rotating night shifts and risk of skin cancer in the nurses' health study. J Natl Cancer Inst 103: 602-606, 2011.

34. Fan L, Sun G, Ma T, Zhong F and Wei W: Melatonin overcomes apoptosis resistance in human hepatocellular carcinoma by targeting survivin and XIAP. J Pineal Res 55: 174-183, 2013.

35. Liu L, Xu Y and Reiter RJ: Melatonin inhibits the proliferation of human osteosarcoma cell line MG-63. Bone 55: 432-438, 2013.

36. Trubiani O, Recchioni R, Moroni F, Pizzicannella J, Caputi S and Di Primio R: Melatonin provokes cell death in human B-lymphoma cells by mitochondrial-dependent apoptotic pathway activation. J Pineal Res 39: 425-431, 2005.

37. Hong Y, Won J, Lee Y, Lee S, Park K, Chang KT and Hong Y: Melatonin treatment induces interplay of apoptosis, autophagy, and senescence in human colorectal cancer cells. J Pineal Res 56 264-274, 2014

38. Jardim-Perassi BV, Arbab AS, Ferreira LC, Borin TF, Varma NR, Iskander AS, Shankar A, Ali MM and de Campos Zuccari DA: Effect of melatonin on tumor growth and angiogenesis in xenograft model of breast cancer. PLoS One 9: e85311, 2014

39. Jung-Hynes B, Schmit TL, Reagan-Shaw SR, Siddiqui IA, Mukhtar H and Ahmad N: Melatonin, a novel Sirtl inhibitor, imparts antiproliferative effects against prostate cancer in vitro in culture and in vivo in TRAMP model. J Pineal Res 50: 140-149, 2011.

40. Paroni R, Terraneo L, Bonomini F, Finati E, Virgili E, Bianciardi P, Favero G, Fraschini F, Reiter RJ, Rezzani R, et al: Antitumour activity of melatonin in a mouse model of human prostate cancer: Relationship with hypoxia signalling. J Pineal Res 57: 43-52, 2014.

41. Rubin BP, Nishijo K, Chen HI, Yi X, Schuetze DP, Pal R, Prajapati SI, Abraham J, Arenkiel BR, Chen QR, et al: Evidence for an unanticipated relationship between undifferentiated pleomorphic sarcoma and embryonal rhabdomyosarcoma. Cancer Cell 19: 177-191, 2011.

42. Borenfreund E and Puerner JA: Toxicity determined in vitro by morphological alterations and neutral red absorption. Toxicol Lett 24: 119-124, 1985.

43. Repetto G, del Peso A and Zurita JL: Neutral red uptake assay for the estimation of cell viability/cytotoxicity. Nat Protoc 3: 1125-1131, 2008.

44. Battistelli M, Salucci S, Burattini S and Falcieri E: Further considerations on in vitro skeletal muscle cell death. Muscles Ligaments Tendons J 3: 267-274, 2013.

45. Salucci S, Burattini S, Battistelli M, Baldassarri V, Curzi D, Valmori A and Falcieri E: Melatonin prevents chemical-induced haemopoietic cell death. Int J Mol Sci 15: 6625-6640, 2014.
46. Oltvai ZN, Milliman CL and Korsmeyer SJ: Bcl-2 heterodimerizes in vivo with a conserved homolog, Bax, that accelerates programmed cell death. Cell 74: 609-619, 1993.

47. Youle RJ and Strasser A: The BCL-2 protein family: Opposing activities that mediate cell death. Nat Rev Mol Cell Biol 9: 47-59, 2008.

48. Chipuk JE, Moldoveanu T, Llambi F, Parsons MJ and Green DR: The BCL-2 family reunion. Mol Cell 37: 299-310, 2010.

49. Zhang S, Zuo L, Gui S, Zhou Q, Wei W and Wang Y: Induction of cell differentiation and promotion of endocan gene expression in stomach cancer by melatonin. Mol Biol Rep 39: 2843-2849, 2012

50. Sainz RM, Mayo JC, Tan DX, León J, Manchester L and Reiter RJ: Melatonin reduces prostate cancer cell growth leading to neuroendocrine differentiation via a receptor and PKA independent mechanism. Prostate 63: 29-43, 2005.

51. Vijayalaxmi, Thomas CR Jr, Reiter RJ and Herman TS: Melatonin: From basic research to cancer treatment clinics. J Clin Oncol 20: 2575-2601, 2002.

52. Panzer A and Viljoen M: The validity of melatonin as an oncostatic agent. J Pineal Res 22: 184-202, 1997.

53. Lissoni $\mathrm{P}$, Barni $\mathrm{S}$, Mandalà $\mathrm{M}$, Ardizzoia $\mathrm{A}$, Paolorossi $\mathrm{F}$, Vaghi M, Longarini R, Malugani F and Tancini G: Decreased toxicity and increased efficacy of cancer chemotherapy using the pineal hormone melatonin in metastatic solid tumour patients with poor clinical status. Eur J Cancer 35: 1688-1692, 1999.

54. Reiter RJ, Tan DX, Sainz RM, Mayo JC and Lopez-Burillo S: Melatonin: Reducing the toxicity and increasing the efficacy of drugs. J Pharm Pharmacol 54: 1299-1321, 2002.

55. Fan LL, Sun GP, Wei W, Wang ZG, Ge L, Fu WZ and Wang H: Melatonin and doxorubicin synergistically induce cell apoptosis in human hepatoma cell lines. World J Gastroenterol 16: $1473-1481,2010$

56. Reiter RJ and Tan DX: What constitutes a physiological concentration of melatonin? J Pineal Res 34: 79-80, 2003.

57. Venegas C, García JA, Escames G, Ortiz F, López A, Doerrier C, García-Corzo L, López LC, Reiter RJ and Acuña-Castroviejo D: Extrapineal melatonin: Analysis of its subcellular distribution and daily fluctuations. J Pineal Res 52: 217-227, 2012.

58. Seabra ML, Bignotto M, Pinto LR Jr and Tufik S: Randomized, double-blind clinical trial, controlled with placebo, of the toxicology of chronic melatonin treatment. J Pineal Res 29: 193-200, 2000.

59. Reiter RJ, Tan DX, Rosales-Corral S and Manchester LC: The universal nature, unequal distribution and antioxidant functions of melatonin and its derivatives. Mini Rev Med Chem 13: 373-384, 2013.

60. Sainz RM, Mayo JC, Rodriguez C, Tan DX, Lopez-Burillo S and Reiter RJ: Melatonin and cell death: Differential actions on apoptosis in normal and cancer cells. Cell Mol Life Sci 60: 1407-1426, 2003

61. Wölfler A, Caluba HC, Abuja PM, Dohr G, Schauenstein K and Liebmann PM: Prooxidant activity of melatonin promotes fas-induced cell death in human leukemic Jurkat cells. FEBS Lett 502: 127-131, 2001.

62. Bizzarri M, Proietti S, Cucina A and Reiter RJ: Molecular mechanisms of the pro-apoptotic actions of melatonin in cancer: A review. Expert Opin Ther Targets 17: 1483-1496, 2013.

63. Galano A, Tan DX and Reiter RJ: On the free radical scavenging activities of melatonin's metabolites, AFMK and AMK. J Pineal Res 54: 245-257, 2013.

64. Bejarano I, Espino J, Barriga C, Reiter RJ, Pariente JA and Rodríguez AB: Pro-oxidant effect of melatonin in tumour leucocytes: Relation with its cytotoxic and pro-apoptotic effects. Basic Clin Pharmacol Toxicol 108: 14-20, 2011

65. Sánchez-Sánchez AM, Martín V, García-Santos G, RodríguezBlanco J, Casado-Zapico S, Suarez-Garnacho S, Antolín I and Rodriguez C: Intracellular redox state as determinant for melatonin antiproliferative vs cytotoxic effects in cancer cells. Free Radic Res 45: 1333-1341, 2011.

66. Büyükavci M, Ozdemir O, Buck S, Stout M, Ravindranath Y and Savaşan S: Melatonin cytotoxicity in human leukemia cells: Relation with its pro-oxidant effect. Fundam Clin Pharmacol 20: 73-79, 2006. 